\title{
HIDROGELES MICROESTRUCTURADOS DE ACRILAMIDA Y DETERMINACIÓN DE SU CAPACIDAD DE ABSORBER AGUA Y ETANOL
}

Jorge Alberto Cortés Ortega*

Departamento de Química Universidad de Guadalajara, Boul. M. García Barragán \# 1451 Guadalajara, Jal. 44430 Mexico

Recebido em 28/3/12; aceito em 14/2/13; publicado na web em 15/4/13

\begin{abstract}
NANOSTRUCTURED HYDROGELS OF ACRYLAMIDE AND DETERMINING THEIR ABILITY TO ABSORB WATER AND ETHANOL. This paper reports the synthesis of nanostructured hydrogels of acrylamide by a two-step polymerisation process. The first step is performed by inverse microemulsion polymerization (water-in-oil) of N-isopropylacrylamide (NIPA), with these particles then added to aqueous solutions of acrylamide (AM), adding a crosslinking agent. The polymerization reaction is then initiated, thereby producing nanostructured hydrogels. We determined the capacity to absorb water, water and / or ethanol when nanostructured hydrogels were immersed in aqueous solutions with different concentrations of ethanol. It was found that the hydrogels were selective in absorbing water in all cases increasing with the ethanol concentration of the solutions.
\end{abstract}

Keywords: acrylamide; ethanol; hydrogels.

\section{INTRODUCCIÓN}

Con la necesidad de nuevos combustibles, se ha estudiado la posibilidad de utilizar etanol o mezclándolo en diferentes proporciones con gasolina. Hoy en día se utilizan tres tipos de materias primas para la producción a gran escala de etanol de origen biológico (bioetanol): Substancias con alto contenido de sacarosa, caña de azúcar, remolacha, melazas, sorgo dulce, Sustancias con alto contenido de almidón, maíz, patata, yuca, Substancias con alto contenido de celulosa, madera, residuos agrícolas, ${ }^{1-6}$ para obtener alcohol que pueda ser utilizado como biocombustible debe de ser purificado mediante procesos de destilación, ${ }^{4,5}$ es por ello que el presente trabajo pretende la purificación del licor de fermentación, mediante el uso hidrogeles de acrilamida que tienen la capacidad de absorber agua y no absorben etanol, ${ }^{7}$ se necesitan hidrogeles que sean capaces de absorber grandes cantidades de agua, para realizar un proceso previo de remoción de agua, logrando incrementar la concentración de etanol de las soluciones acuosas, por lo que la síntesis de hidrogeles de acrilamida superabsorbentes, es de gran importancia, para incrementar la concentración de etanol en el licor resultante de la fermentación y disminuir los costos de obtención. Los hidrogeles preparados a partir de acrilamida son capaces de absorber entre 15 a 40 gramos de agua por cada gramo de xerogel, dependiendo de la cantidad de agua y de agente entrecruzante presente en la mezcla inicial de la reacción. ${ }^{7,8}$ Se ha encontrado que la adición de nanoparticulas a matrices poliméricas, incrementa la capacidad de absorber agua de estos materiales hasta en un $250 \% .^{7-9}$ Por lo que en este trabajo se sintetizaron hidrogeles microestructurados de acrilamida, con partículas de n-isopropilacrilamida, estos hidrogeles fueron preparados en dos etapas, en la primera de ellas las partículas fueron sintetizadas mediante reacción de polimerización en microemulsión inversa, en una segunda etapa se prepararon soluciones acuosas al 10\% de acrilamida, adicionando las partículas, con $1 \%$ de agente entrecruzante, iniciador y acelerador con respecto de la carga total, estos hidrogeles fueron colocados en soluciones acuosas de etanol, para determinar su capacidad de remover el agua de las soluciones acuosas, para incrementar la concentración de etanol en la solución residual.

\section{PARTE EXPERIMENTAL}

\section{Materiales y reactivos}

Los monómeros empleados en la síntesis de los hidrogeles fueron la N-isopropilacrilamida (NIPA) con una pureza de $99 \%$ estabilizada con MEHQ, proveniente de Tokyo Kasei, La acrilamida (AM), con una pureza del $99 \%$ proveniente de Merck. Como iniciador se utilizó persulfato de potasio con una pureza del $99 \%$ y el acelerador fue $\mathrm{N}, \mathrm{N}, \mathrm{N}^{\prime}, \mathrm{N}^{\prime}$-tetrametil-etilendiamina (TMEDA) de Tokyo Kasei El agente entrecruzante fue $\mathrm{N}^{\prime}, \mathrm{N}^{\prime}$-metilenbisacrilamida (NMBA) con una pureza de $99 \%$ de Tokyo Kasei. Como surfactante se utilizó Aerosol-OT (di-(2-etilhexil)sulfosuccianato de Sodio) (AOT) con $98 \%$ de pureza de TCI América. Los solventes utilizados en las microemulsiones inversas fueron tolueno con una pureza de $99 \%$ de Lancaster, y agua bi-destilada de Productos Selectropura.

\section{Preparación de partículas}

La reacción de polimerización se llevo a cabo a $25{ }^{\circ} \mathrm{C}$, con la siguiente composición de Tolueno/AOT/Solución acuosa de NIPA al 20\% (28/14/20), Primero se coloco el AOT y el tolueno a agitar durante 30 min., al cabo de este tiempo se adiciono la solución acuosa de NIPA, con $1 \%$ (en peso) de agente entrecruzante, a los 5 min. se adiciono $1 \%$ de iniciador, y $1 \%$ de acelerador con respecto del monómero. Dejándose reaccionar por 4 hr. Todas las partículas fueron preparadas con el procedimiento anterior, variando la cantidad de tolueno en la mezcla inicial de reacción, las composiciones de los diferentes látex sintetizados, son presentados en la tabla 1.

Tabla 1. Composición de la mezcla inicial de reacción de los látex preparados

\begin{tabular}{ccccc}
\hline Muestra & Tolueno (gr.) & AOT (gr.) & NIPA (gr.) & AGUA (gr.) \\
\hline L1a & 28 & 14 & 4 & 16 \\
L1aa & 40 & 14 & 4 & 16 \\
L1ab & 48 & 14 & 4 & 16 \\
L1ac & 52 & 14 & 4 & 16 \\
L1ad & 60 & 14 & 4 & 16 \\
L1ae & 64 & 14 & 4 & 16 \\
\hline
\end{tabular}




\section{Medición del tamaño de partícula}

Para la determinación de 1 tamaño de partícula se utilizó el dispersor cuasielastica de luz marca Zetasizer NANO ZS90, este proceso se realizó dos formas diferentes: la primera medición se realizo directamente del látex obtenido una vez que la reacción de polimerización ha terminado. Para realizar la segunda medición el látex fue secado totalmente, el material seco obtenido (el cual contiene AOT, partículas de poliNIPA, poliNIPA no adicionada a la partícula y NIPA que no reacciono) fue disuelto en agua, esta solución fue preparada para que tenga $1 \%$ de poliNIPA (estableciendo que toda la NIPA adicionada a la mezcla inicial haya reaccionado), esta composición fue escogida, ya que cuando se preparen los hidrogeles microeestructurados, será dicha composición la que se necesite para obtener soluciones acuosas al 10\% de monómeros con una composición de acrilamida/ partículas de 90/10.

\section{SÍNTESIS DE HIDROGELES MICROESTRUCTURADOS}

La síntesis de los hidrogles microestructurados se realizo mediante dos procedimientos:

\section{1.- Proceso 1 (Utilización directa del látex)}

En este proceso se utilizo directamente el látex obtenido de la reacción de polimerización en microemulsión. Para ello se calculo la cantidad necesaria de látex (que contiene las partículas de n-isopropilacrilamida, el tolueno y el AOT), acrilamida y agua para preparar soluciones acuosas conteniendo $10 \%$ de monómeros (acrilamida y partículas), con $1 \%$ de agente entrecruzante con respecto de los monómeros y $90 \%$ de solventes (agua y tolueno), posteriormente se adicionó $1 \%$ de iniciador y $1 \%$ de acelerador con respecto de los monómeros. Cuando se forma la mezcla se coloca en recipientes de vidrio, se tapan y se sellan con papel parafilm, dejándose reaccionar por un día a temperatura ambiente. Estas muestras se identifican como AM/L1A, AM/L1Aa, AM/L1Ab...

\section{2.- Proceso 2 (utilización del material seco)}

En este proceso el látex obtenido en la reacción de polimerización en microemulsión se coloco a secar, tres días a temperatura ambiente y posteriormente en una estufa de vació a $45^{\circ} \mathrm{C}$ hasta peso constante. Posteriormente se calculo la cantidad necesaria de material seco (que contiene las partículas y el AOT) para preparar soluciones acuosas conteniendo $10 \%$ de monómeros (acrilamida y partículas), con $1 \%$ de agente etrecruzante y $90 \%$ de agua, posteriormente se adiciona $1 \%$ de iniciador y $1 \%$ de acelerador con respecto de los monómeros, esta solución se coloca en recipientes de vidrio, los cuales se tapan y se sellan con papel parafilm. Dejándose reaccionar por un día a temperatura ambiente. Estas mustras se identifican como: AM/L1As, AM/L1Aas, AM/L1Abs ...

\section{Proceso de limpieza}

Transcurrido el tiempo de reacción, se remueven los geles del recipiente y se colocan a secar, una vez secos se pesan y se introducen en agua destilada, a $25^{\circ} \mathrm{C}$ por una semana, para permitir que las muestras alcancen el hinchamiento de equilibrio, posteriormente se colocan en un baño de temperatura controlada a $45^{\circ} \mathrm{C}$ por tres días. Al termino de este periodo se cambia el agua de lavado; continuando con este ciclo hasta que al adicionar al agua de lavado, una solución fría de $\mathrm{CaCl}_{2}$, no se forme turbiedad, la cual aparece por la formación del compuesto insoluble $\mathrm{Ca}(\mathrm{AOT})_{2}$, en trabajos previos se ha encontrado que al seguir este procedimiento se logra un buen proceso de limpieza. ${ }^{9,10}$
Una vez terminado el proceso de limpieza, las muestras se colocan a secar, tres días a temperatura ambiente (esto se realizo para evitar que la evaporación brusca del agua fracture las muestras), posteriormente en una estufa de vacío a $45^{\circ} \mathrm{C}$, hasta peso constante, con el peso inicial y el peso final se determina el rendimiento de la reacción.

\section{Cinética de hinchamiento}

Las muestras totalmente secas, se pesan y se colocaron en agua bidestilada a $25{ }^{\circ} \mathrm{C}$ para determinar su cinética de hinchamiento. Esto se realizo pesando las muestras a diferentes tiempos, secando su superficie con papel absorbente. De la diferencia entre el peso de la muestra seca y el peso de la muestra hinchada se calcula el hinchamiento con la siguiente ecuación:

$$
H=\frac{\text { peso húmedo }- \text { peso } \operatorname{seco}}{\text { peso } \operatorname{seco}}=\frac{p h-p s}{p s}
$$

Al graficar el hinchamiento contra el tiempo se obtiene la cinética de hinchamiento. Este proceso se repitió colocando las muestras en etanol absoluto (Figura 1).
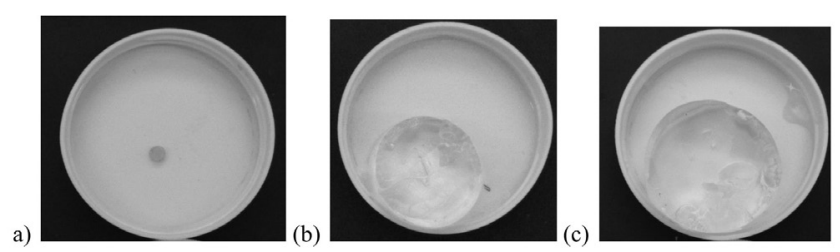

Figura 1. Se presenta la evolución en el hinchamiento de la muestras L1Aas en las primeras $48 \mathrm{hr}$. del experimento, xerogel tiempo cero (a), $24 \mathrm{hr}$. (b) y $48 \mathrm{hr}$

\section{Medición de la capacidad de extraer agua de soluciones acuosas de etanol}

Se selecciono la muestra L1A para determinar la capacidad de absorber agua de soluciones acuosas de etanol, modificando la concentración de etanol desde $20 \%$ y hasta $80 \%$ y con una relación de 300 gramos de solución por cada gramo de xerogel; dejándose llegar al hinchamiento de equilibrio (el tiempo en el que se alcanza el equilibrio es determinado por la cinética de hinchamiento obtenida con el procedimiento anterior). Con el peso del gel hinchado, la masa residual de la solución y su índice de refracción (determinado por un refractómetro digital Reichert AR200) se pudo determinar la cantidad de agua y etanol absorbido por las muestras.

\section{Análisis morfológico de los hidrogeles sintetizados}

La preparación de la muestras para ser observadas bajo el microscopio electrónico de barrido Hitachi TM-100 fue: Se colocan las muestras en agua destilada, cuando alcanzan el hinchamiento de equilibrio se sumergen en etanol absoluto, con lo que las muestras se deshidratan adquiriendo un color blanco lechoso como se muestra en la Figura 2.

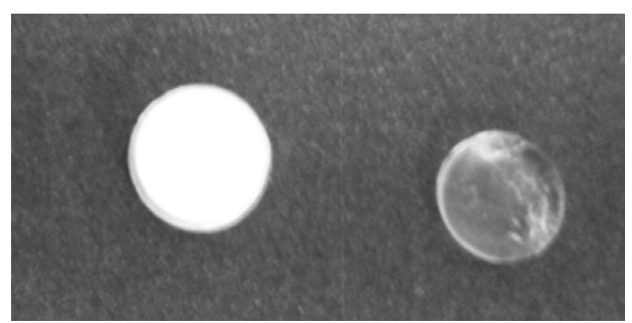

Figura 2. Se presenta el aspecto físico de los hidrogeles deshidratados en etanol absoluto y el xerogel 

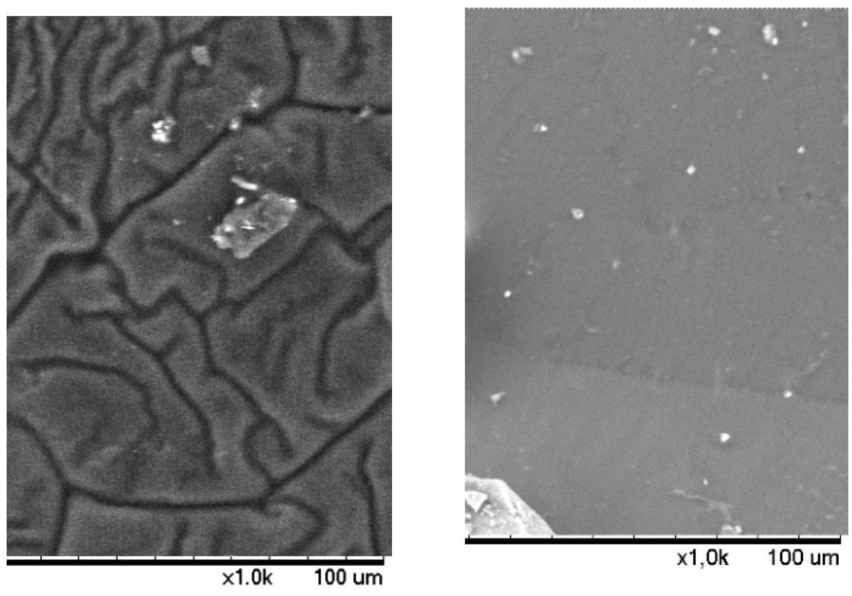

Figura 3. Fotografías de las muestras de hidrogel convencional y microestructurado tomadas con el microscopio electrónico de barrido Hitachi TM-100, al ser sometidas al proceso de deshidratación

\section{DISCUSIÓN Y RESULTADOS}

\section{Tamaño de partícula}

En la tabla 2 podemos observar un comportamiento errático en los tamaños de partícula medidos directamente de los diferentes látex producidos por las reacciones de polimerización, lo cual resulta previsible, ya que al analizar la composición del tolueno en las diferentes composiciones de reacción, esta composición inicia con un $45 \%$ de tiolueno y termina con $65 \%$, con lo cual cambia la viscosidad del medio. Y teniendo en cuenta que el equipo se calibra en función de la viscosidad del medio, la lectura del tamaño de partícula obtenido es poco confiable.

Tabla 2. Se presentan los tamaños de partícula, obtenidos de las reacciones de polimerización

\begin{tabular}{ccc}
\hline Muestra & $\begin{array}{c}\text { Tamaño de partícula medido } \\
\text { directamente del látex nm }\end{array}$ & $\begin{array}{c}\text { Tamaño de partícula de la } \\
\text { redispersión en agua } \mathrm{nm}\end{array}$ \\
\hline L1a & $25 \pm 5$ & $388 \pm 10$ \\
L1aa & $83 \pm 8$ & $457 \pm 15$ \\
L1ab & $69 \pm 9$ & $690 \pm 10$ \\
L1ac & $29 \pm 5$ & $826 \pm 11$ \\
L1ad & $23 \pm 7$ & $827 \pm 12$ \\
L1ae & $62 \pm 6$ & $1564 \pm 25$ \\
\hline
\end{tabular}

En tanto que cuando el látex se somete al proceso de secado y el material obtenido es redispersado en agua, se puede observar que el tamaño de partícula obtenido, aumenta con el incremento en la concentración de tolueno en el látex, ya que al existir mayor concentración de solvente (en la mezcla de reacción) las partículas en crecimiento se encuentran mas alejadas unas de otras, por lo que tienen mayor espacio y las cadenas en formación dentro de la partícula crecen con mayor libertad existiendo una menor proporción de entrecruzamientos físicos, ${ }^{11}$ lo que permite un mayor diámetro de partícula en las miscelas formadas. Esto mismo ha sido encontrado en polímeros sintetizados en solución acuosa. ${ }^{11}$

\section{Cinética de hinchamiento}

Las líneas sólidas en la Figura 4 son las predicciones del modelo de hinchamiento de segundo orden (muestra AM/L1Aa), el cual es comúnmente usado para modelar las cinéticas de hinchamiento. ${ }^{12}$ Este modelo tiene la siguiente forma:

$$
\frac{d W}{d t}=K\left(W_{\infty}-W\right)^{2}
$$

donde:

$$
W=\frac{\text { peso húmedo }- \text { peso } \text { seco }}{\text { peso húmedo }}=\frac{p h-p s}{p h}
$$

que al ser integrado con $\mathrm{W}=0 \mathrm{y} \mathrm{t}=0$ se obtiene:

$$
\frac{t}{W}=\frac{1}{k W_{\infty}^{2}}+\frac{t}{W_{\infty}}
$$

al combinar las ecuaciones 1 y 3 se obtienen las ecuaciones 5 y 6 :

$$
\begin{gathered}
W=\frac{H}{H+1} \\
W=\frac{H_{\infty}}{H_{\infty}+1}
\end{gathered}
$$

al sustituirse 5 y 6 en 4 se obtiene:

$$
\frac{t(H+1)}{H}=\frac{\left(H_{\infty}+1\right)^{2}}{K H_{\infty}^{2}}+\frac{H_{\infty}+1}{H_{\infty}} t
$$

al graficar $\mathrm{t}(\mathrm{H}+1) / \mathrm{H}$ vs el tiempo se obtiene una línea recta donde:

$$
\begin{array}{ll}
\text { la pendiente es: } & m=\frac{H_{\infty}+1}{H_{\infty}} t \\
\text { el intercepto } & b=\frac{\left(H_{\infty}+1\right)^{2}}{K H_{\infty}^{2}}
\end{array}
$$

y el valor de $\mathrm{K}$ se puede calcular con:

$$
K=\frac{\left(H_{\infty}+1\right)^{2}}{b H_{\infty}^{2}}
$$

esta ecuación se puede reacomodar como:

$$
H=\frac{t}{t(m-1)-b}
$$

Al utilizar la ecuación (11) se puede obtener el hinchamiento en función de la temperatura en la muestra de AM/L1Aa (90/10), en la Figura 4 la línea sólida representa la predicción teórica y los símbolos son los datos experimentales. Los valores de la desviación estándar y el coeficiente de regresión $\mathrm{R}$ (tablas 3 y 4) muestran que el modelo ajusta con buena aproximación los datos experimentales, este modelo ha sido aplicado por satisfactoriamente en otros trabajos de investigación. ${ }^{9,10,12}$

En la tabla 3 se puede observar un incremento en la capacidad de absorber agua a $25^{\circ} \mathrm{C}$, para todos los hidrogeles microestructurados, cuando se compara con los hidrogeles convencionales. Se puede observar que las muestras que fueron preparadas directamente con el látex, incrementan su capacidad de absorber agua, cuando se compara con la muestra de poliAM, pero esta capacidad es menor, cuando de compara con la capacidad de absorber agua, que presentan las muestras preparadas con la adición del látex seco.

Esto se debe a que en las muestras sintetizadas mediante la adición directa del látex, para lograr que las partículas tengan una 


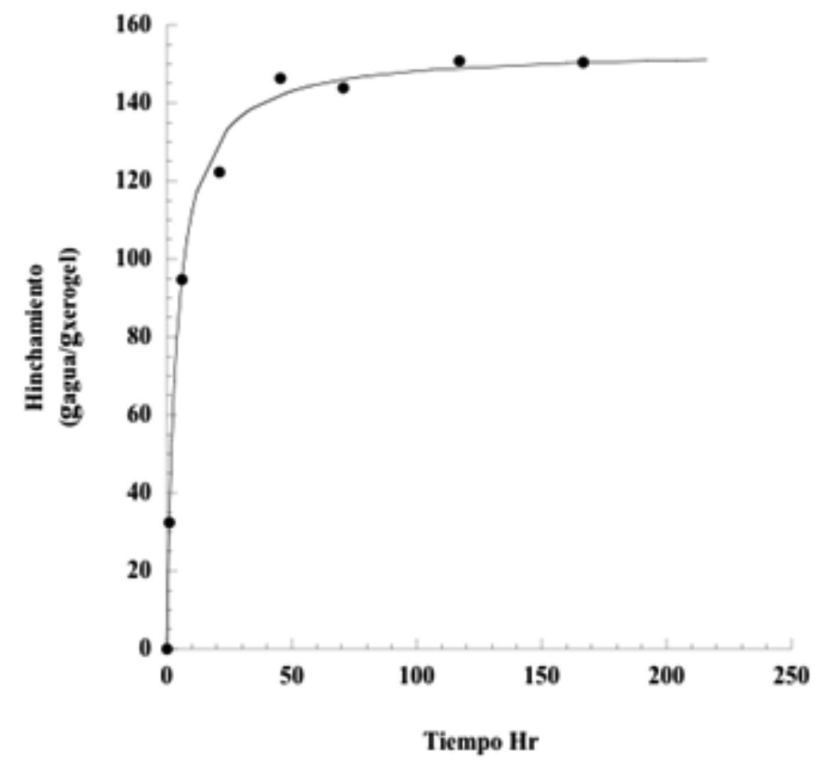

Figura 4. Cinética de hinchamiento de hidrogeles microestrcuturados de acrilamida, datos experimentales (-) y datos teóricos obtenidos con la ecuación 3 (-)

distribución lo mas homogénea posible, se adiciona de forma simultanea el iniciador, el acelerador y el látex, para que la reacción se lleve lo mas rápido posible, lo cual no permite que las partículas alcancen su hinchamiento de equilibrio, con lo cual quedan con un mayor grado de rigidez dentro de la matriz, ${ }^{11}$ por lo que disminuye su contribución a la capacidad de absorber agua de los hidrogeles.

En tanto que cuando el látex se somete al proceso de secado y el sólido resultante que contiene partículas de NIPA y AOT, se somete al proceso de redispersión (el cual tarda alrededor de 25 min. con agitación continua) permitiendo que el agua difunda hacia las partículas, las cuales alcanzan el hinchamiento de equilibrio cuando se forma la red; ${ }^{13}$ lo que incrementa la cantidad de agua que pueden absorber las partículas y por lo tanto la red polimérica.

En trabajos previos se ha visto que la adición de nanoparticulas tanto hidrófilas como hidrófobas incrementa la capacidad de absorber agua de los hidrogeles, ${ }^{7,8}$ estos trabajos establecieron que aunque ambas partículas propiciaban un incremento en la capacidad de absorber agua de los hidrogeles. Las partículas hidrófilas no solo incrementaban la capacidad de absorber agua, por los huecos formados dentro de la red, si no que estas partículas también contribuyen absorbiendo agua. ${ }^{7}$ Las formas que se pueden apreciar en la figura 3 se deben al colapsamiento de estos huecos formados y de las partículas, como las muestras son sometidas a la deshidratación brusca mediante su inmersión en etanol absoluto, cuando el gel colapsa los huecos forman la arrugas de forma definidas del hidrogel microestructurado, en tanto que en el hidrogel convencional dichas formas no se aprecian.

La uniformidad de la capacidad de absorber agua de los hidrogeles fue estudiada al colocar las partes extremas y central del cilindro formado en la síntesis de los hidrogeles, obteniéndose desviaciones estándar máximas del 5\% en todas las muestras reportadas.

Así mismo se determino la influencia del AOT adicionado a los hidrogeles, tanto por la adición del látex como de la mezcla seca, se puede observar en la tabla 3, que la cantidad de agua que absorbe el hidrogel de AM que se preparo con AOT, es ligeramente mayor que el hidrogel de AM pura, en trabajos previos se observo que el contacto entre soluciones acuosas de AOT con polidimetilacrilamida provoca la aparición de dos fases dentro de una solución lamellar, ${ }^{13}$ provocando que cuando se forme la red polimérica, se obtenga un gel mas heterogéneo, con mayores huecos que en la poliaAM, incrementando su capacidad de absorber agua, por lo que ambos factores, (la adición del AOT y las partículas de poliNIPA) contribuyen a incrementar la capacidad de absorber agua de los hidrogeles microestructurados.

\section{Capacidad de absorber soluciones de etanol/agua}

Se escogió la muestra L1As, para evaluar la capacidad de absorber agua de la muestra en función de la concentración de etanol en soluciones acuosas, en la tabla 5 se puede observar como disminuye la capacidad de absorber agua de los hidrogeles cuando la concentración de etanol se incrementa, hasta que en composiciones mayores de $50 \%$ prácticamente no absorben agua, a cada solución en la cual se sumergió el hidrogel, se le midio la concentración de etanol mediante el uso del refractómetro, y en todos los casos las lecturas indicaron que el etanol no penetra la red y solo se remueve agua. Asi mismo se puede observar el incremento en la concentración de etanol de la solución.

\section{Reversibilidad de la capacidad de absorber agua de los hidrogeles colocados en soluciones acuosas de etano}

Una vez que los hidrogeles alcanzaron su hinchamiento de equilibrio, en las soluciones acuosas de etanol, se colocaron a secar hasta peso constante, enseguida fue comparado el peso seco final

Tabla 3. Hinchamiento de equilibrio de hidrogeles microestructurados de acrilamida preparados directamente con el látex

\begin{tabular}{cccccccccc}
\hline & AM/L1a & AM/L1aa & AM/L1ab & AM/L1ac & AM/L1ad & AM/L1ae & AM/AOT & AM \\
\hline $\mathrm{m}$ & 1.017 & 1.024 & 1.023 & 1.021 & 1.018 & 1.022 & 1.058 & 1.060 \\
$\mathrm{~b}$ & 18.714 & 27.714 & 23.720 & 31.388 & 12.836 & 25.147 & 7.564 & 28.305 \\
$\mathrm{H}_{\infty}$ & 59.910 & 42.162 & 43.377 & 47.296 & 54.930 & 45.076 & 17.184 & 16.613 \\
$\mathrm{~K}$ & 0.055 & 0.038 & 0.044 & 0.033 & 0.081 & 0.042 & 0.148 & 0.040 \\
desv est & 0.414 & 0.370 & 0.523 & 0.495 & 0.772 & 0.482 & 0.392 & 0.388 \\
\hline
\end{tabular}

Tabla 4. Hinchamiento de equilibrio de hidrogeles microestructurados de acrilamida preparados con el material seco

\begin{tabular}{|c|c|c|c|c|c|c|}
\hline & $\mathrm{AM} / \mathrm{L} 1 \mathrm{as}$ & $\mathrm{AM} / \mathrm{L} 1 \mathrm{aas}$ & $\mathrm{AM} / \mathrm{L} 1 \mathrm{abs}$ & AM/L1acs & AM/L1ads & AM/L1aes \\
\hline $\mathrm{m}$ & 1.007 & 1.006 & 1.004 & 1.004 & 1.004 & 1.007 \\
\hline $\mathrm{b}$ & 1.453 & 1.374 & 4.162 & 2.440 & 0.702 & 4.439 \\
\hline $\mathrm{H}_{\infty}$ & 153.723 & 160.212 & 276.913 & 260.852 & 252.181 & 152.745 \\
\hline $\mathrm{K}$ & 0.697 & 0.737 & 0.242 & 0.413 & 1.435 & 0.228 \\
\hline desv est & 2.086 & 3.104 & 6.580 & 6.292 & 4.337 & 3.199 \\
\hline
\end{tabular}


Tabla 5. Hinchamiento de equilibrio de la muestra AM/L1As en soluciones acuosas de etanol

\begin{tabular}{ccccc}
\hline $\begin{array}{c}\text { Composición } \\
\text { inicial }\end{array}$ & $\begin{array}{c}\text { Composición } \\
\text { final }\end{array}$ & $\begin{array}{c}\text { Masa de solu- } \\
\text { ción inicial }\end{array}$ & $\begin{array}{c}\text { Masa de agua } \\
\text { removida }\end{array}$ & $\begin{array}{c}\text { Masa de solu- } \\
\text { ción residual }\end{array}$ \\
\hline $20 \%$ & $33.52 \%$ & 300 & 121.01 & 178.99 \\
$40 \%$ & $56.50 \%$ & 300 & 87.59 & 212.41 \\
$50 \%$ & $50.20 \%$ & 300 & 1.21 & 298.79 \\
$60 \%$ & $60.15 \%$ & 300 & 0.73 & 299.27 \\
$80 \%$ & $80.02 \%$ & 300 & 0.07 & 299.93 \\
$100 \%$ & $100 \%$ & 300 & 0.00 & 300.00 \\
\hline
\end{tabular}

con el peso seco inicial, esto con el fin de determinar la cantidad de materia perdida durante el proceso, encontrándose que en todo los casos la perdida de masa de las muestras se encuentran en intervalos menores al $0,5 \%$. Posteriormente estas muestras fueron colocadas de nuevo en las soluciones acuosas de etanol para verificar la reversibilidad de la capacidad de absorber agua. Se encontró que en todos los casos la desviación estándar de la capacidad de absorber agua de las muestras se encontraba dentro del $5 \%$. Este proceso fue realizado en tres ocasiones repitiéndose los resultados observados y descritos anteriormente.

\section{CONCLUSIONES}

En el presente trabajo se encontró que cuando se mide el tamaño de partícula en el látex obtenido (de la reacción de polimerización en microemulsión inversa), se podría especular que el tamaño de partícula no se ve afectado en gran medida, por las modificaciones en el contenido de tolueno, pero cuando se mide el tamaño de partícula de los nanopolímeros en agua, después del proceso de secado y redispersión; se pudo determinar que la concentración de tolueno en la microemulsión tiene gran influencia en el tamaño final de las partículas; el tamaño de las partículas obtenido determina en gran medida la capacidad de absorber agua de los hidrogeles microestructurados, sintetizados en la segunda etapa. Se observo que se obtienen mejores resultados en la capacidad de absorber agua de los hidrogeles microestructurados, cuando las partículas son adicionadas secas, que cuando el látex se adiciona directamente a este proceso de polimerización. Se determino la capacidad de remover agua de las soluciones acuosas de etanol, obteniéndose buenos resultados ya que se pudo incrementar la concentración de etanol, en mas de 10 puntos porcentuales.

\section{AGRADECIMIENTOS}

Se agradece al financiamiento de PROMEP mediante el proyecto identificado UDG-PTC-492

\section{REFERENCIAS}

1. Arifeen N.; Wang R.; Kookos I.; Webb C.; and Koutinas A. A.; Appl. Microbiol. Biotechnol. 2007, 23, 872.

2. Laluce C.; Tognolli J. O.; de Oliveira K. F.; Serra Souza C. and Rezende Morais M.; Appl. Microbiol. Biotechnol. 2009, 83, 627.

3. Alam M. Z.; Kabbashi N. A.; and Razak A. A.; Bioresource Technol. 2008, 99, 4709.

4. Polycarpou P.; Renewable Energy. 2009, 34, 2525.

5. Najafi G.; Ghobadian B.; Tavakoli T.; and Yusaf T.; Renewable Sustainable Energy Rev. 2009, 13, 1418.

6. Rodríguez L. A.; Toro M.E.; Vazquez F.; Correa-Daneri M. L.; Gouiric S. C.; and Vallejo M.D.; Int. J. Hydrogen Energy. 2010, 35, 5165.

7. Puig, L. J.; Sanchez-Daz, J.C.; Villacampa, M.; Mendizabal, E.; Puig, J.E.; Aguiar, A.; Katime, I.; J. Colloid Interface Sci. 2001, 235, 278.

8. Cortes J. A.; Doctoral Thesis, Universidad de Guadalajara, México, 2005.

9. Nuño-Donlucas S. M., Sánchez-Díaz J. C., Rabelero M., Cortés-Ortega J., Luhrs-Olmos C. C.; Fernández-Escamilla V. V.; Mendizábal E.; Puig J. E.; J. Colloid Interface Sci. 2004, 270, 94.

10. Cortés J. A.; Puig J. E.; Morales J. A.; y Mendizábal E.; Rev. Mex. Ing. Quim. 2011, 10, 513.

11. Hirai M.; Kawai-Hirai R.; Sanada M.; Iwase H.; and Mitsuya S.; J. Phys. Chem. B 1999, 103, 9658 .

12. Katime I.; Velada J. L.; Novoa R.; Diaz de Apodaca E.; Puig J. y Mendizábal E.; Polym. Int. 1996, 40, 281.

13. Pacios I.E.; Renamayor C.S.; Horta A.; Lindman B.; and Thuresson K.; J. Phys. Chem. B. 2002, 106, 5035. 